\title{
3-Aminopyrroles and their application in the synthesis of pyrrolo[3,2-d]pyrimidine (9-deazapurine) derivatives
}

\author{
Abdellatif M. Salaheldin,* Ana M. F. Oliveira-Campos, and Lígia M. Rodrigues \\ Centro de Química, IBQF, Universidade do Minho, Campus de Gualtar, 4710-057 Braga, \\ Portugal \\ E-mail: salaheldin@quimica.uminho.pt
}

\begin{abstract}
3-Aminopyrrole derivatives have been synthesized from 3-anilino-2-cyanoacrylonitrile using Thorpe-Ziegler cyclization. These substituted pyrroles are readily converted into $5 H$-pyrrolo[3,2$d]$ pyrimidine (9-deazapurines).
\end{abstract}

Keywords: 3 -Aminopyrrole, pyrrolo[3,2- $d]$ pyrimidine, 9-deazapurines, $\beta$-enaminonitriles, Thorpe-Ziegler cyclization

\section{Introduction}

Polyfunctional derivatives of 3-aminopyrroles constitute an important family of compounds due to their wide applications as antibacterial, antiviral, anticonvulsant, anti-inflammatory, analgesic, and antipyretic activities. ${ }^{1-4}$ Due to the importance of pyrrole for various applications, great efforts have been made towards the preparation of this heterocyclic system. ${ }^{5-9}$ Recently we described several efficient approaches to heteroaromatic systems using functionally substituted enamine precursors. ${ }^{10-14}$

In continuation to our interest in the chemistry of $\beta, \beta$-enaminonitriles we report here the results of our work aimed at exploring the potential utility of 3-anilino-2-cyanoacrylonitrile in the heterocyclic synthesis. The synthesized $\beta, \beta$-enaminonitriles $\mathbf{2}$ are converted into the corresponding 3-aminopyrrole derivatives by reaction with $\alpha$-haloketones under basic conditions, a Thorpe-Ziegler cyclization. ${ }^{15,16}$ These substituted pyrroles are readily converted to $5 H_{-}$ pyrrolo[3,2-d]pyrimidines (9-deazapurines). ${ }^{17-19}$ 


\section{Results and Discussion}

$\beta, \beta$-Enaminonitrile $\mathbf{2}$ was readily synthesized by the reaction of ethoxymethylene malononitrile $\mathbf{1}$ with $p$-anisidine in ethanol at room temperature. ${ }^{20}$ Enaminonitriles 2 were found to be good candidates to obtain 3-aminopyrrole based on a Thorpe-Ziegler cyclization. ${ }^{15,16}$ In this method, $N$-alkylation of a $\beta, \beta$-enaminonitrile was carried out using $\alpha$-haloketones in anhydrous DMF in the presence of $\mathrm{K}_{2} \mathrm{CO}_{3}$ as the base. Moreover, compounds having an aryl substituent on the amino moiety of the enamine group were the most convenient for alkylation and spontaneous intramolecular cyclization. The presence of this group facilitates the formation of the $\mathrm{N}$-anion required for alkylation and subsequent carbanion formation for the cyclization involving the cyano group.

The reaction of enaminonitrile 2 with chloroacetonitrile, chloroacetone, ethyl bromoacetate and $\alpha$-bromoacetophenone in $\mathrm{DMF} / \mathrm{K}_{2} \mathrm{CO}_{3}$ afforded the corresponding 3-aminopyrrole derivatives $\mathbf{4 a - d}$ in low yield (24-45\%) via intermediate $\mathbf{3}$. We prepared compound $\mathbf{4}$ by a modification of the method used by Gewald et al. ${ }^{15,16}$ using triethylamine as alkaline reagent. When the reaction was carried out in an excess of triethylamine solution, the desired 3aminopyrrole derivatives 4a-d are obtained in a satisfactory yield (74-91\%).

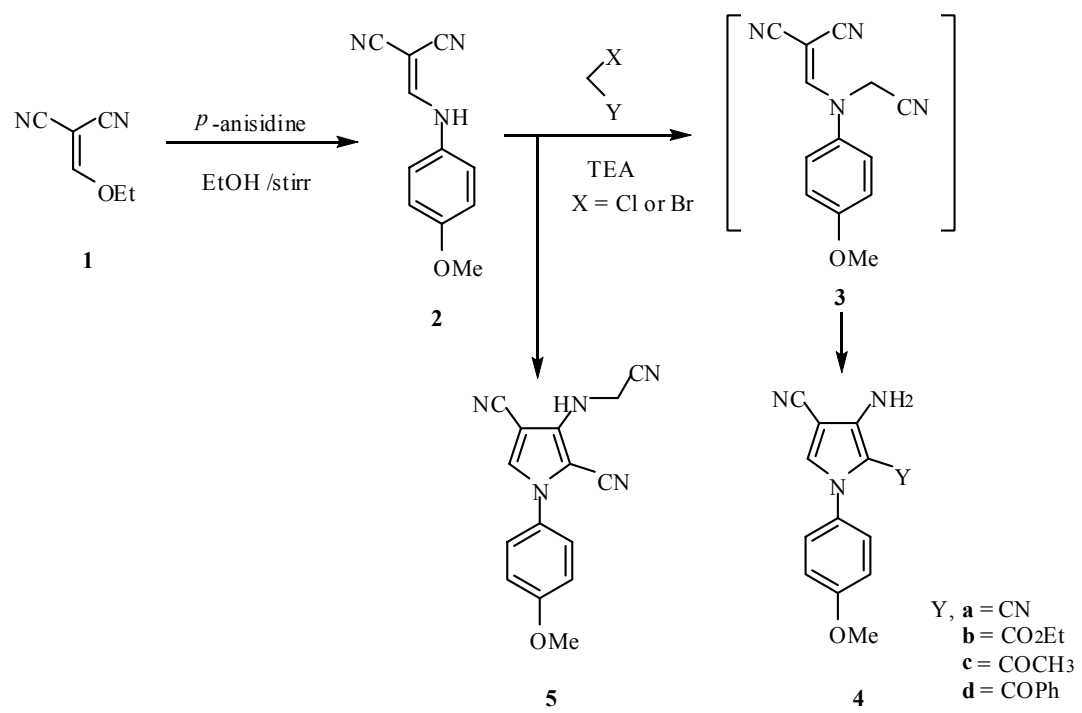

\section{Scheme 1}

The structure of compounds 4a-d was established on the basis of elemental analysis, IR, mass, ${ }^{1} \mathrm{H}$ and ${ }^{13} \mathrm{C}$ NMR spectral data studies (cf. Experimental Section). For example, the ${ }^{1} \mathrm{H}$ NMR spectrum of compound 4a showed the absence of a signal for a methylene function and the presence of a two protons $\mathrm{D}_{2} \mathrm{O}$ exchangeable signal at $\delta=6.11 \mathrm{ppm}$ for the amino function and a singlet for the pyrrole 5 -H proton at $\delta=7.84 \mathrm{ppm} .{ }^{13} \mathrm{C}$ NMR and mass spectra of compound $\mathbf{4 a}$ 
are in agreement with the proposed structure. When the reaction was carried out in excess of chloroacetonitrile, the $N$-substituted product 5 was obtained (Scheme 1).

The 3-amino-2-cyanopyrrole $\mathbf{4 a}$ is a polyfunctional compound containing an interesting set of substituents. Although the 3-amino group has an electronegative substituent in the neighboring ortho position, it retains its basic properties and is readily acylated in refluxing acetic anhydride to afford the monoacetyl derivative $\mathbf{6}$.

The most generally used approach to pyrrolo[3,2- $d]$ pyrimidines has so far involved elaboration of the pyrrole ring onto a preformed pyrimidine bearing reactive functionalities at C-4 and C-5. ${ }^{18,19,21}$ Another strategy has involved the formation of the pyrimidine ring onto a preformed 3-aminopyrrole intermediate ${ }^{22}$ as we describe herein.

Compound 4a,b reacted with triethyl orthoformate or dimethyl formamide dimethylacetal (DMFDMA) to give the corresponding imidocarboxylate derivatives $\mathbf{7}$ and amidine derivatives $\mathbf{8}$ respectively, which are the key compounds for the preparation of pyrrolo[3,2- $d]$ pyrimidine derivatives. Thus, compound $\mathbf{7 a}$ was stirred at room temperature in methanolic ammonia to produce 4-aminopyrrolopyrimidine 9 and not the isomeric form 10 (Scheme 2). Attempts to obtain 9 from 8 a according to literature methods ${ }^{17}$ failed. The identity of pyrrolopyrimidine 9 was confirmed by ${ }^{1} \mathrm{H}$ NMR, NOE experiment and elemental analysis. Thus, the presence or absence of an Nuclear Overhauser Enhancement between specific protons allowed establishing the structure of compound 9.

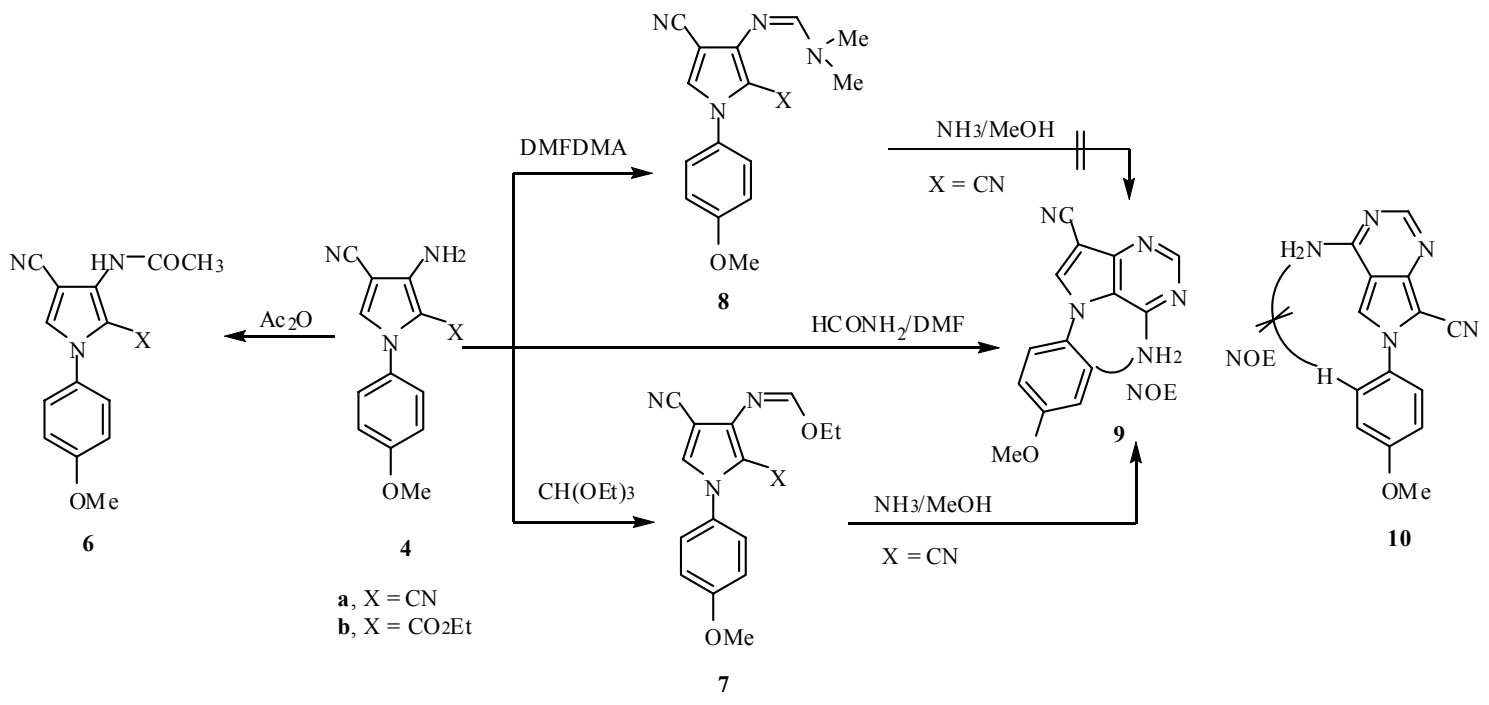

\section{Scheme 2}

Compound 9 was also obtained on heating compound $4 \mathbf{a}$ in a mixture of $\mathrm{HCO}_{2} \mathrm{H} / \mathrm{HCONH}_{2} / \mathrm{DMF}$. 
Following the behavior that was observed with ammonia solution, compounds 7a and 8a reacted with aniline under reflux to produce the Dimroth rearrangement product, 4-substituted aminopyrrolopyrimidine 12, via the intermediate 11 (Scheme 3). ${ }^{14,23}$

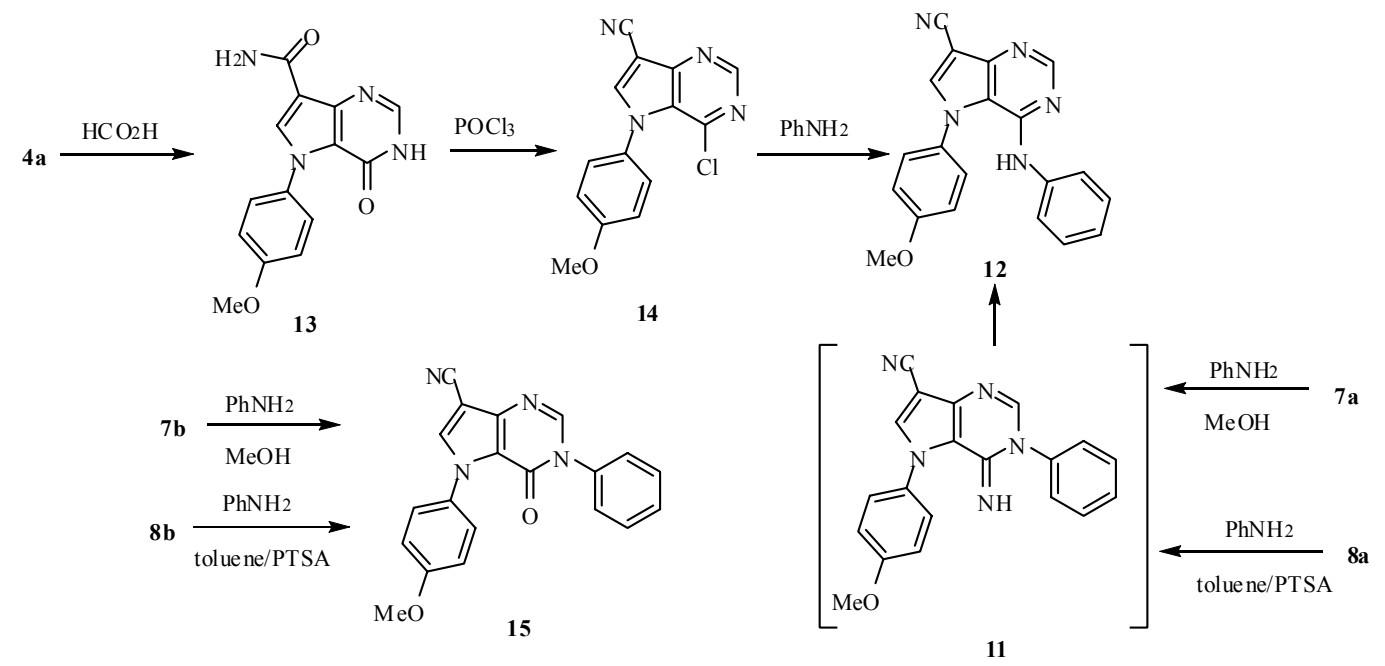

\section{Scheme 3}

Based on NMR data the alternative structure 11 was excluded. The ${ }^{1} \mathrm{H}$ NMR spectra of compound 12 showed all the expected signals for aromatic protons and four singlets signals at $\delta$ $=3.86\left(\mathrm{OCH}_{3}\right), 6.98(\mathrm{NH}), 8.52(2-\mathrm{H})$ and $8.62(6-\mathrm{H})$, which was not sufficient to differentiate between structures 11 and 12. For this reason, we obtained the HMQC and HMBC NMR spectra and made an unambiguous assignment in the ${ }^{1} \mathrm{H}$ and ${ }^{13} \mathrm{C}$ NMR spectrum (see Experimental Section).

In the HMBC spectrum, we observe an intense correlation peak for the NH proton at $\delta=6.98$ with the carbons peak at $\delta=120.68 \mathrm{ppm}\left(\mathrm{C}-2^{\prime \prime}, 6^{\prime \prime}\right)$, which is characteristic only for structure 12 but not for 11, where the indicated proton and carbon atoms are separated by five bonds.

To confirm the structure of compound $\mathbf{1 2}$ an independent route was followed, compound $\mathbf{4 a}$ was refluxed in boiling formic acid to produce pyrrolopyrimidinone-7-carboxamide 13. Compound 13 was refluxed in phosphorus oxychloride to obtain 4-chloropyrrolopyrimidines $14^{24}$, then reacting compound 14 with aniline, the pyrrolo[3,2-d]pyrimidine 12 (65\%) was isolated, whose spectral characteristics were completely coincident with the samples obtained before (Scheme 3 ).

Under the same reaction conditions, the amidines $\mathbf{7 b}$ and $\mathbf{8 b}$ reacted with aniline to afford the bicyclic compound 4-oxo-3-phenylpyrrolo[3,2-d]pyrimidine 15. Also in these cases the ester group in position 2 of the pyrrole ring but not the 4-cyano group participates in the cyclization, as shown in the ${ }^{1} \mathrm{H}$ NMR spectra which revealed the absence of the ester groups (no triplet and quartet signals are found) and the presence of CN signal in the IR at $2232 \mathrm{~cm}^{-1}$ and at $\delta 117.02$ ppm in the ${ }^{13} \mathrm{C}$ NMR spectra. 


\section{Experimental Section}

General Procedures. Melting points were determined on a Gallenkamp melting point apparatus and are uncorrected. IR spectra were registered on a Perkin Elmer FTIR-1600 using Nujol emulsions between $\mathrm{NaCl}$ plates. ${ }^{1} \mathrm{H}$ NMR (300 or $400 \mathrm{MHz}$ ) and ${ }^{13} \mathrm{C}$ NMR $(75.4$ or $100.62 \mathrm{MHz})$ spectra were recorded in deuterated dimethylsulfoxide $\left[\mathrm{D}_{6}\right] \mathrm{DMSO}$ or deuterated chloroform $\mathrm{CDCl}_{3}$ on a Varian Unity Plus Spectrometer using tetramethylsilane (TMS) as an internal reference, and results are expressed as $\delta$ values. Double resonance, HMQC and HMBC experiments were carried out for complete assignment of proton and carbon signals in the NMR spectra, whenever possible. Mass spectra were performed on a Shimadzu GCMS-QP 1000 Ex mass spectrometer at $70 \mathrm{eV}$. Elemental analyses were obtained on a Leco CHNS-932 instrument. Compound $\mathbf{2}$ was prepared according to the literature. ${ }^{20}$

\section{General procedure for preparation of 3-aminopyrrole derivatives 4a-d}

Method A. A mixture of $2(0.01 \mathrm{~mol})$, the $\alpha$-halo compound (chloroacetonitrile, chloroacetone, ethyl bromoacetate and $\alpha$-bromoacetophenone $(0.011 \mathrm{~mol})$, and potassium carbonate $(2.0 \mathrm{~g})$ in dimethylformamide $(20 \mathrm{~mL})$ was stirred for $1 \mathrm{~h}$, at $90{ }^{\circ} \mathrm{C}$, in an oil-bath. The reaction mixture was cooled and poured into water $(60 \mathrm{~mL})$. The precipitated solid products formed were filtered off, washed thouroughly with cold water and recrystallized from EtOH to afford the corresponding cyclized products $4 \mathbf{a}(45 \%), \mathbf{4 b}(24 \%), \mathbf{4 c}(39 \%), \mathbf{2 d}(35 \%)$.

Method B. To a solution of the intermediate $2(0.01 \mathrm{~mol})$ the $\alpha$-halo compound (chloroacetonitrile, chloroacetone, ethyl bromoacetate and $\alpha$-bromoacetophenone $)(0.011 \mathrm{~mol})$ and triethylamine $(4 \mathrm{~mL})$ were added with external cooling. The reaction mixture was refluxed for 10-15 minutes, after cooling (50 mL) water was added, the solid product was filtered off, washed thoroughly with cold water and crystallized from ethanol (in case of $\mathbf{4 a}, 91 \%$ ). For derivatives $\mathbf{4 b}$-c a brown oil was separated, the water was decanted and the oil was extracted with $\mathrm{CH}_{2} \mathrm{Cl}_{2}(3 \times 25 \mathrm{~mL})$ and the combined organic layers were dried $\left(\mathrm{Na}_{2} \mathrm{SO}_{4}\right)$, filtered and the solvent was evaporated to give a solid which was crystallized from EtOH.

3-Amino-1-(4-methoxyphenyl)-1 $\boldsymbol{H}$-pyrrole-2,4-dicarbonitrile (4a). Yield (91\%), beige solid, mp. 186-188 ${ }^{\circ} \mathrm{C}$ (EtOH) [Lit. Mp. 187-188] ${ }^{15}$; IR (Nujol): $v=3437,3349\left(\mathrm{NH}_{2}\right), 2227,2205(\mathrm{CN})$ $\mathrm{cm}^{-1} ;{ }^{1} \mathrm{H}$ NMR (DMSO-d $\left.)_{6}\right): \delta=3.80\left(\mathrm{~s}, 3 \mathrm{H}, \mathrm{OCH}_{3}\right), 6.11\left(\mathrm{~s}, 2 \mathrm{H}, \mathrm{NH}_{2}\right), 7.07$ (d, $2 \mathrm{H}, J=8.8 \mathrm{~Hz}$, $\left.\mathrm{H}^{\prime} 3^{\prime}, 5^{\prime}\right), 7.41$ (d, $\left.2 \mathrm{H}, J=8.8 \mathrm{~Hz}, \mathrm{H}-2^{\prime}, 6^{\prime}\right), 7.84$ (s, 1H, H-5); ${ }^{13} \mathrm{C}$ NMR (DMSO-d 6 ): $\delta=55.56$ $\left(\mathrm{OCH}_{3}\right), 83.23(\mathrm{C}-4), 88.18(\mathrm{C}-2), 113.38(\mathrm{CN}), 114.23(\mathrm{CN}), 114.71\left(\mathrm{C}-3^{\prime}, 5^{\prime}\right), 125.44\left(\mathrm{C}-2^{\prime}, 6^{\prime}\right)$, 130.12 (C-1'), 132.52 (C-5), 148.32 (C-3), 159.21 (C-4'). Anal. Calcd. for $\mathrm{C}_{13} \mathrm{H}_{10} \mathrm{~N}_{4} \mathrm{O}$ (238.24): C, 65.54; H, 4.23; N, 23.52. Found: C, 65.44; H, 4.28; N, 23.44.

Ethyl 3-amino-4-cyano-1-(4-methoxyphenyl)-1 H-pyrrole-2-carboxylate (4b). Yield (74\%), white solid, mp. 163-164 ${ }^{\circ} \mathrm{C}$ (EtOH) [Lit. Mp. 159-161] ${ }^{15}$; IR (Nujol): $v=3443,3343\left(\mathrm{NH}_{2}\right)$, $2215(\mathrm{CN}), 1657(\mathrm{CO}) \mathrm{cm}^{-1}$; ${ }^{1} \mathrm{H}$ NMR (DMSO-d $\left.{ }_{6}\right): \delta=0.98$ (t, $\left.3 \mathrm{H}, J=7.5 \mathrm{~Hz}, \mathrm{CH}_{3}\right), 3.78(\mathrm{~s}, 3 \mathrm{H}$, $\mathrm{OCH}_{3}$ ), 3.99 (q, $2 \mathrm{H}, J=7.5 \mathrm{~Hz}, \mathrm{CH}_{2}$ ), 5.93 (s, 2H, $\mathrm{NH}_{2}$ ), 6.95 (d, 2H, $J=8.7 \mathrm{~Hz}, \mathrm{H}-3{ }^{\prime}, 5$ ) ), 7.24 (d, $\left.2 \mathrm{H}, J=9.0 \mathrm{~Hz}, \mathrm{H}-2{ }^{\prime}, 6{ }^{\prime}\right), 7.65$ (s, $\left.1 \mathrm{H}, \mathrm{H}-5\right) ;{ }^{13} \mathrm{C}$ NMR (DMSO-d 6 ): $\delta=13.96\left(\mathrm{CH}_{3}\right), 55.45$ 
$\left(\mathrm{OCH}_{3}\right), 59.00\left(\mathrm{CH}_{2}\right), 83.26(\mathrm{C}-4), 105.72(\mathrm{C}-2), 113.53\left(\mathrm{C}-3^{`}, 5^{`}\right), 114.80(\mathrm{CN}), 127.18\left(\mathrm{C}^{\prime}\right)^{\prime}$, 6'), 132.58 (C-1`), 134.46 (C-5), 146.36 (C-3), 158.76 (C-4'), 160.03 (CO). Anal. Calcd. for $\mathrm{C}_{15} \mathrm{H}_{15} \mathrm{~N}_{3} \mathrm{O}_{3}$ (285.30): C, 63.15; H, 5.30; N, 14.73. Found: C, 63.46; H, 5.38; N, 14.43.

5-Acetyl-4-amino-1-(4-methoxyphenyl)-1H-pyrrole-3-carbonitrile (4c). Yield (78\%), white solid, mp. 212-214 ${ }^{\circ} \mathrm{C}(\mathrm{EtOH})$; IR $(\mathrm{KBr}): v=3404,3289\left(\mathrm{NH}_{2}\right), 2222(\mathrm{CN}), 1688(\mathrm{C}=\mathrm{O}) \mathrm{cm}^{-1}$; ${ }^{1} \mathrm{H}$ NMR $\left(\mathrm{DMSO}_{6}\right): \delta=1.63\left(\mathrm{~s}, 3 \mathrm{H}, \mathrm{CH}_{3}\right), 3.80\left(\mathrm{~s}, 3 \mathrm{H}, \mathrm{OCH}_{3}\right), 6.68\left(\mathrm{~s}, 2 \mathrm{H}, \mathrm{NH}_{2}\right), 7.04(\mathrm{~d}, 2 \mathrm{H}$, $\left.J=9 \mathrm{~Hz}, \mathrm{H}-3^{`}, 5^{`}\right), 7.38$ (d, 2H, $\left.J=9 \mathrm{~Hz}, \mathrm{H}-2^{`}, 6^{`}\right), 7.66$ (s, 1H, H-5); ${ }^{13} \mathrm{C}$ NMR (DMSO-d 6$): \delta$ $=28.54\left(\mathrm{CH}_{3}\right), 55.53\left(\mathrm{OCH}_{3}\right), 81.59(\mathrm{C}-4), 114.48\left(\mathrm{C}-3^{`}, 5^{`}\right), 114.68(\mathrm{CN}), 118.21(\mathrm{C}-2), 127.93$ (C-2`,6 ), 132.21 (C-1'), 135.21 (C-5), 147.45 (C-3), 159.38 (C-4'), 186.16 (CO). Anal. Calcd. for $\mathrm{C}_{14} \mathrm{H}_{13} \mathrm{~N}_{3} \mathrm{O}_{2}$ (255.27): C, 65.87; H, 5.13; N, 16.46. Found: C, 65.88; H, 4.94; N, 16.49 .

4-Amino-5-benzoyl-1-(4-methoxyphenyl)-1 $\boldsymbol{H}$-pyrrole-3-carbonitrile (4d). Yield (80\%), pale yellow solid, mp. $187-188^{\circ} \mathrm{C}(\mathrm{EtOH})$; IR (Nujol): $v=3423,3318\left(\mathrm{NH}_{2}\right), 2220(\mathrm{CN}), 1678(\mathrm{CO})$ $\mathrm{cm}^{-1} ;{ }^{1} \mathrm{H}$ NMR $\left(\mathrm{DMSO}_{\mathrm{d}}\right): \delta=3.61\left(\mathrm{~s}, 3 \mathrm{H}, \mathrm{OCH}_{3}\right), 6.58\left(\mathrm{~s}, 2 \mathrm{H}, \mathrm{NH}_{2}\right), 6.64(\mathrm{~d}, 2 \mathrm{H}, J=9 \mathrm{~Hz}$, Ar-H), 7.02 (d, 2H, J=9 Hz, Ar-H), 7.08-7.22 (m, 5H, Ar-H), 7.83 (s, 1H, H-5). Anal. Calcd. for $\mathrm{C}_{19} \mathrm{H}_{15} \mathrm{~N}_{3} \mathrm{O}_{2}$ (317.34): C, 71.91; H, 4.76; N, 13.24. Found: C, 71.78; H, 5.14; N, 13.11.

3-(Cyanomethylamino)-1-(4-methoxyphenyl)-1H-pyrrole-2,4-dicarbonitrile (5). This compound was prepared similarly to the method B used above by using 2 equivalents of chloroacetonitrile. Yield (55\%); pale yellow solid, mp. 198-200 ${ }^{\circ} \mathrm{C}(\mathrm{EtOH})$; IR (Nujol): $v=3333$ $(\mathrm{NH}), 2224,2203(\mathrm{CN}) \mathrm{cm}^{-1} ;{ }^{1} \mathrm{H}$ NMR (DMSO-d $): \delta=3.81\left(\mathrm{~s}, 3 \mathrm{H}, \mathrm{OCH}_{3}\right), 4.35(\mathrm{~d}, 2 \mathrm{H}, J=7$ $\left.\mathrm{Hz}, \mathrm{CH}_{2}\right), 7.10$ (d, 2H, $\left.J=9 \mathrm{~Hz}, \mathrm{H}-3^{`}, 5^{\prime}\right), 7.13$ (s, 1H, NH), 7.47 (d, 2H, $\left.J=9 \mathrm{~Hz}, \mathrm{H}-2^{`}, 6^{\prime}\right)$, $8.05(\mathrm{~s}, 1 \mathrm{H}, \mathrm{H}-5) ;{ }^{13} \mathrm{C}$ NMR $\left(\mathrm{DMSO}_{6}\right): \delta=32.78\left(\mathrm{CH}_{2}\right), 55.61\left(\mathrm{OCH}_{3}\right), 83.37(\mathrm{C}-4), 89.13(\mathrm{C}-$ 2), $113.18(\mathrm{CN}), 113.76(\mathrm{CN}), 114.76\left(\mathrm{C}^{\prime} 3^{`}, 5^{`}\right), 117.99(\mathrm{CN}), 126.08$ (C-2`,6 ), $129.65\left(\mathrm{C}^{\prime} 1^{\prime}\right)$, 133.92 (C-5), 145.42 (C-3), 159.58 (C-4).- MS (EI, $70 \mathrm{eV}): m / z(\%)=237.08(34), 250.08$ (100), 277.09 (36, M+).

Anal. Calcd. for $\mathrm{C}_{15} \mathrm{H}_{11} \mathrm{~N}_{5} \mathrm{O}$ (277.28): C, 64.97; H, 4.00; N, 25.26. Found: C 64.87, H 4.16, N 24.95 .

\section{Reaction with acetic anhydride}

To compound 4a,b $(0.01 \mathrm{~mol})$ was added acetic anhydride $(10 \mathrm{~mL})$, the reaction mixture was heated under reflux for $3 \mathrm{~h}$, cooled and the precipitate was filtered off.

$\mathbf{N}$-(2,4-Dicyano-1-(4-methoxyphenyl)-1H-pyrrol-3-yl)acetamide (6a). Yield: (72\%); pale yellow solid, mp. 229-230 ${ }^{\circ} \mathrm{C}(\mathrm{EtOH})$. IR (Nujol): $v=320(\mathrm{NH}), 2236,2226(\mathrm{CN}), 1671(\mathrm{CO})$ $\mathrm{cm}^{-1}$; ${ }^{1} \mathrm{H}$ NMR (DMSO-d $): \delta=2.09\left(\mathrm{~s}, 3 \mathrm{H}, \mathrm{CH}_{3}\right), 3.82\left(\mathrm{~s}, 3 \mathrm{H}, \mathrm{OCH}_{3}\right), 7.12(\mathrm{~d}, 2 \mathrm{H}, J=8.7 \mathrm{~Hz}$, Ar-H), 7.52 (d, 2H, $J=9.0 \mathrm{~Hz}, \mathrm{Ar}-\mathrm{H}), 8.21$ (s, 1H, H-5), 10.46 (s, 1H, NH). MS (EI, $70 \mathrm{eV}): \mathrm{m} / z$ $(\%)=280.09(\mathrm{M}+, 6) ; 238.08$ (100); 223.06 (32); 195.09 (14).

Anal. Calcd. for $\mathrm{C}_{15} \mathrm{H}_{12} \mathrm{~N}_{4} \mathrm{O}_{2}$ (280.096): C, 64.28; H, 4.32; N, 19.99. Found: C, 64.03; H, 4.30; N, 19.92.

Ethyl 3-acetamido-4-cyano-1-(4-methoxyphenyl)-1H-pyrrole-2-carboxylate (6b).

Yield (75\%); white solid, mp. 240-241 ${ }^{\circ} \mathrm{C}(\mathrm{AcOH})$. IR (Nujol): $v=3237(\mathrm{NH}) ; 2238(\mathrm{CN}) ; 1714$, $1672(\mathrm{CO}) \mathrm{cm}^{-1} ;{ }^{1} \mathrm{H}$ NMR (DMSO-d 6$): \delta=1.04\left(\mathrm{t}, 3 \mathrm{H}, J=7.5 \mathrm{~Hz}, \mathrm{CH}_{3}\right), 2.05\left(\mathrm{~s}, 3 \mathrm{H}, \mathrm{CH}_{3}\right), 3.79$ 
$\left(\mathrm{s}, 3 \mathrm{H}, \mathrm{OCH}_{3}\right), 4.03$ (q, 2H, $\left.J=7.5 \mathrm{~Hz}, \mathrm{CH}_{2}\right), 6.99$ (d, 2H, $\left.J=6.9 \mathrm{~Hz}, \mathrm{Ar}-\mathrm{H}\right), 7.29$ (d, 2H, $J=6.8$ $\mathrm{Hz}, \mathrm{Ar}-\mathrm{H}), 7.93$ (s, 1H, H-5), 9.75 (s, 1H, NH). Anal. Calcd. for $\mathrm{C}_{17} \mathrm{H}_{17} \mathrm{~N}_{3} \mathrm{O}_{4}$ (327.33): C, 62.38; H, 5.23; N, 12.84. Found: C, 62.25; H, 5.02; N, 12.80 .

\section{Reaction with triethyl orthoformate $(7 \mathbf{a}, \mathbf{b})$}

A mixture of 3-aminopyrrole-4-carbonitrile, $4 \mathbf{a}, \mathbf{b}(0.015 \mathrm{~mol})$, triethyl orthoformate $(20 \mathrm{~mL})$ was heated under reflux for $7 \mathrm{~h}$ and then evaporated under reduced pressure. The residue was treated with ethanol and the solid product formed was collected by filtration, washed with ethanol and crystallized from EtOH.

Ethyl $\mathbf{N}$-2,4-dicyano-1-(4-methoxyphenyl)-1 $H$-pyrrol-3-ylimidoformate (7a). Yield (84\%); white solid, mp. $164-165{ }^{\circ} \mathrm{C}(\mathrm{EtOH})$; IR (Nujol): $v=2233,2215(\mathrm{CN}), 1639(\mathrm{C}=\mathrm{N}) \mathrm{cm}^{-1} ;{ }^{1} \mathrm{H}$ NMR (DMSO-d $\left.)_{6}\right): \delta=1.32\left(\mathrm{t}, 3 \mathrm{H}, J=7.5 \mathrm{~Hz}, \mathrm{CH}_{3}\right), 3.82\left(\mathrm{~s}, 3 \mathrm{H}, \mathrm{OCH}_{3}\right), 4.31$ (q, $2 \mathrm{H}, J=7.5 \mathrm{~Hz}$, $\left.\mathrm{CH}_{2}\right), 7.12(\mathrm{~d}, 2 \mathrm{H}, J=9.0 \mathrm{~Hz}, \mathrm{Ar}-\mathrm{H}), 7.52$ (d, 2H, $\left.J=9.0 \mathrm{~Hz}, \mathrm{Ar}-\mathrm{H}\right), 8.19(\mathrm{~s}, 1 \mathrm{H}, \mathrm{N}=\mathrm{CH}), 8.33$ (s, 1H, H-5). - MS (EI, $70 \mathrm{eV}): m / z(\%)=294.11$ (58, M+); 266.08 (26); 238.08 (100); 223.06 (78). Anal. Calcd. for $\mathrm{C}_{16} \mathrm{H}_{14} \mathrm{~N}_{4} \mathrm{O}_{2}$ (294.31): C, 65.30; H, 4.79; N, 19.04. Found: C, 65.74; $\mathrm{H}$, $4.64 ; \mathrm{N}, 18.95$.

Ethyl 4-cyano-3- (ethoxymethylenamino)-1- (4-methoxyphenyl)-1H- pyrrole-2-carboxylate (7b). Yield (82\%); white solid, mp. 171-172 ${ }^{\circ} \mathrm{C}(\mathrm{EtOH})$; IR (Nujol): $v=2228$ (CN), 1703 (CO), $1640(\mathrm{C}=\mathrm{N}) \mathrm{cm}^{-1} ;{ }^{1} \mathrm{H}$ NMR $\left(\mathrm{DMSO}_{-}\right): \delta=1.00\left(\mathrm{t}, 3 \mathrm{H}, J=7.5 \mathrm{~Hz}, \mathrm{CH}_{3}\right), 1.32(\mathrm{t}, 3 \mathrm{H}, J=7.5$ $\mathrm{Hz}, \mathrm{CH}_{3}$ ), 3.79 (s, 3H, $\mathrm{OCH}_{3}$ ), 3.96 (q, 2H, $\left.J=7.5 \mathrm{~Hz}, \mathrm{CH}_{2}\right), 4.29$ (q, 2H, J=7.5 Hz, $\mathrm{CH}_{2}$ ), 6.98 $(\mathrm{d}, 2 \mathrm{H}, J=9.0 \mathrm{~Hz}, \mathrm{Ar}-\mathrm{H}), 7.27$ (d, 2H, $J=9.0 \mathrm{~Hz}, \mathrm{Ar}-\mathrm{H}), 7.89$ (s, 1H, N=CH), 8.03 (s, 1H, H-5). Anal. Calcd for $\mathrm{C}_{18} \mathrm{H}_{19} \mathrm{~N}_{3} \mathrm{O}_{4}$ (341.36): C, 63.33; H, 5.61; N, 12.31. Found C, 63.17; H, 5.41; N, 12.34 .

\section{Reaction with DMFDMA}

DMFDMA (0.012 mol) was added to a solution of 3-aminopyrrole-4-carbonitrile 4a,b (0.01 mol), in dry toluene $(40 \mathrm{~mL})$ and the mixture heated under reflux for $7 \mathrm{~h}$. the reaction mixture was cooled and the precipitated solid was filtered off and crystallized from $\mathrm{EtOH}$.

$\boldsymbol{N}^{\prime}$-(2,4-Dicyano-1-(4-methoxyphenyl)-1 $H$-pyrrol-3-yl)- $\boldsymbol{N}, \boldsymbol{N}$-dimethyl imioformamide (8a). Yield (84\%); beige solid, mp. 171-172 ${ }^{\circ} \mathrm{C}(\mathrm{EtOH})$; IR (Nujol): $v=2233,2210(\mathrm{CN}), 1640(\mathrm{C}=\mathrm{N})$ $\mathrm{cm}^{-1} ;{ }^{1} \mathrm{H}$ NMR (DMSO-d $): \delta=3.09\left(\mathrm{~s}, 6 \mathrm{H}, 2 \mathrm{CH}_{3}\right), 3.87\left(\mathrm{~s}, 3 \mathrm{H}, \mathrm{OCH}_{3}\right), 7.01(\mathrm{~d}, 2 \mathrm{H}, J=7.2 \mathrm{~Hz}$, Ar-H), 7.23 (s, 1H, N=CH), 7.34 (d, 2H, $J=6.9 \mathrm{~Hz}, \mathrm{Ar}-\mathrm{H}), 8.04$ (s, 1H, H-5). Anal. Calcd. for $\mathrm{C}_{16} \mathrm{H}_{15} \mathrm{~N}_{5} \mathrm{O}$ (293.32): C, 65.52; H, 5.15; N, 23.88. Found: C, 65.18; H, 5.00; N, 23.95.

Ethyl 4-cyano-3-((dimethylamino)methylenamino)-1-(4-methoxyphenyl)-1H-pyrrole-2carboxylate (8b). Yield (71\%); colorless crystals, mp. 122-123 ${ }^{\circ} \mathrm{C}(\mathrm{EtOH})$; IR (Nujol): v = 2212 $(\mathrm{CN}), 1686(\mathrm{CO}), 1630(\mathrm{C}=\mathrm{N}) \mathrm{cm}^{-1} ;{ }^{1} \mathrm{H}$ NMR (DMSO-d $): \delta=0.97\left(\mathrm{t}, 3 \mathrm{H}, J=7.5 \mathrm{~Hz}, \mathrm{CH}_{3}\right)$, 2.93 (s, 3H, $\left.\mathrm{CH}_{3}\right), 3.00$ (s, 3H, $\left.\mathrm{CH}_{3}\right), 3.78\left(\mathrm{~s}, 3 \mathrm{H}, \mathrm{OCH}_{3}\right), 3.92$ (q, 2H, J=7.5 Hz, $\left.\mathrm{CH}_{2}\right), 6.96$ (d, $2 \mathrm{H}, J=9 \mathrm{~Hz}, \mathrm{Ar}-\mathrm{H}), 7.22$ (d, 2H, $J=9.0 \mathrm{~Hz}, \mathrm{Ar}-\mathrm{H}), 7.70$ (s, 1H, N=CH), 7.75 (s, 1H, H-5). Anal. Calcd. for $\mathrm{C}_{18} \mathrm{H}_{20} \mathrm{~N}_{4} \mathrm{O}_{3}$ (340.38): C, 63.52; H, 5.92; N, 16.46. Found: C, 63.31; H, 5.83; N, 16.40. 


\section{4-Amino-5-(4-methoxyphenyl)-5H-pyrrolo[3,2-d]pyrimidine-7-carbonitrile (9)}

Method A. Amidine $7 \mathbf{a}(2.0 \mathrm{mmol})$ was added to a mixture of methanol $(15 \mathrm{~mL})$ and $25 \%$ aqueous ammonia solution $(15 \mathrm{~mL})$. The reaction mixture was stirred for $3 \mathrm{~h}$, cooled, and the precipitated solid was filtered off and crystallized from EtOH-DMF, 2:1.

Method B. A mixture of 3-amino-2-cyanopyrrole 4a $(0.01 \mathrm{~mol})$, formamide $(15 \mathrm{~mL})$, DMF (5 $\mathrm{mL})$, and formic acid $(2 \mathrm{~mL})$ was heated under reflux for $6-8 \mathrm{~h}$. The reaction mixture was allowed to stand overnight at room temperature. The solid obtained was filtered, washed with cold methanol, dried, and crystallized from EtOH-DMF, 2:1. Yield (74\%); colorless crystals, mp. 281-282 ${ }^{\circ} \mathrm{C}$ (EtOH-DMF); IR (Nujol): $v=3400,3293\left(\mathrm{NH}_{2}\right), 2224(\mathrm{CN}) \mathrm{cm}^{-1}$; ${ }^{1} \mathrm{H}$ NMR $\left(\mathrm{DMSO}_{-} \mathrm{d}_{6}\right): \delta=3.84\left(\mathrm{~s}, 3 \mathrm{H}, \mathrm{OCH}_{3}\right), 6.03$ (brs, $\left.2 \mathrm{H}, \mathrm{NH}_{2}\right), 7.14$ (d, 2H, $\left.J=9.0 \mathrm{~Hz}, \mathrm{H}-3^{`}, 5^{\prime}\right), 7.52$ $\left(\mathrm{d}, 2 \mathrm{H}, J=9.0 \mathrm{~Hz}, \mathrm{H}-2{ }^{\prime}, 6^{\prime}\right), 8.29(\mathrm{~s}, 1 \mathrm{H}, \mathrm{H}-2), 8.46$ (s, 1H, H-6); ${ }^{13} \mathrm{C}$ NMR (DMSO-d 6 ): $\delta=$ $55.61\left(\mathrm{OCH}_{3}\right), 86.44(\mathrm{C}-7), 113.64(\mathrm{C}-7 \mathrm{a}), 114.39(\mathrm{CN}), 114.86\left(\mathrm{C}-3^{`}, 5^{\prime}\right), 127.81\left(\mathrm{C}-2^{\prime}, 6^{\prime}\right)$, 129.945 (C-1'), 139.64 (C-6), 148.38 (C-4a), 150.76 (C-4), 152.63 (C-2), 159.88 (C-4).- MS $(\mathrm{EI}, 70 \mathrm{eV}): \mathrm{m} / \mathrm{z}(\%)=265.09$ (100); 266.10 (13); 264.09 (40); 250.07 (50). Anal. Calcd. for $\mathrm{C}_{14} \mathrm{H}_{11} \mathrm{~N}_{5} \mathrm{O}$ (265.27): C, 63.39; H, 4.18; N, 26.40. Found: C, 63.48; H, 3.99; N, 26.12.

\section{5-(4-Methoxyphenyl)-4-(phenylamino)-5H-pyrrolo[3,2-d]pyrimidine-7-carbonitrile (12)}

Method A. To a solution of amidine $7 \mathbf{a}(3.0 \mathrm{mmol})$ in methanol $(20 \mathrm{~mL})$ was added aniline $(3.0$ mmol). The reaction mixture was heated under reflux for $7 \mathrm{~h}$. The precipitate formed after cooling overnight was filtered off and dried and recrystallized from ethanol (82\%).

Method B. Aniline $(2.0 \mathrm{mmol})$ and $p$-toluenesulfonic acid $(0.01 \mathrm{~g})$ were added to a solution of amidine 8a $(2.0 \mathrm{mmol})$ in toluene $(40 \mathrm{~mL})$. The reaction mixture was refluxed for $7 \mathrm{~h}$, and the precipitated solid was filtered off and crystallized from EtOH (77 \%).

Method C. To a solution of 4-chloropyrrolopyrimidine $14(2.0 \mathrm{mmol})$ in methanol $(20 \mathrm{~mL}) \mathrm{was}$ added aniline $(2.0 \mathrm{mmol})$. The reaction mixture was refluxed for $5 \mathrm{~h}$. The precipitate formed during reflux was filtered off and found identical in all respect with that obtained from method A $(65 \%)$.

Yield (77\%); colorless crystals, mp. 225-226 ${ }^{\circ} \mathrm{C}$ (EtOH-DMF); IR (Nujol): $v=3216(\mathrm{NH}), 2228$ $(\mathrm{CN}) \mathrm{cm}^{-1} ;{ }^{1} \mathrm{H}$ NMR $\left(\mathrm{DMSO}_{-}\right): \delta=3.86\left(\mathrm{~s}, 3 \mathrm{H}, \mathrm{OCH}_{3}\right), 6.98(\mathrm{~s}, 1 \mathrm{H}, \mathrm{NH}), 7.03(\mathrm{t}, 1 \mathrm{H}, J=7.5$ Hz, H-4' ) 7.18 (d, 2H, J=8.7 Hz, H-3', 5'), 7.28 (t, 2H, $J=7.5$ Hz, H-3' ', 5' ), 7.33 (d, 2H, $J$ $\left.=8.7 \mathrm{~Hz}, \mathrm{H}-2^{\prime \prime}, 6^{\prime \prime}\right), 7.65\left(\mathrm{~d}, 2 \mathrm{H}, J=8.7 \mathrm{~Hz}, \mathrm{H}-2^{\prime}, 6^{\prime}\right), 8.52(\mathrm{~s}, 1 \mathrm{H}, \mathrm{H}-2), 8.62(\mathrm{~s}, 1 \mathrm{H}, \mathrm{H}-6) ;{ }^{13} \mathrm{C}$ NMR (DMSO-d $\left.{ }_{6}\right): \delta=55.75\left(\mathrm{OCH}_{3}\right), 86.91(\mathrm{C}-7), 114.11(\mathrm{C}-7 \mathrm{a}), 114.14(\mathrm{CN}), 114.84\left(\mathrm{C}-3^{\prime}, 5^{\prime}\right)$, 120.69 (C-2"', 6"'), 123.42 (C-4"'), 128.06 (C-2',6'), 128.76 (C-3"', 5'), 129.84 (C-1'), 138.35 (C-1' ), 140.49 (C-6), 147.39 (C-4), 148.78 (C-4a), 151.94 (C-2), 160.04 (C-4').- MS (EI, 70 eV): $m / z(\%)=342.13(8) ; 341.12(46, \mathrm{M}+) ; 340.12(100) ; 325.10$ (13); 297.10 (13). Anal. Calcd. for $\mathrm{C}_{20} \mathrm{H}_{15} \mathrm{~N}_{5} \mathrm{O}$ (341.37): C, 70.37; H, 4.43; N, 20.52. Found: C, 70.42; H, 4.36; N, 20.63.

\section{5-(4-methoxyphenyl)-4-oxo-4,5-dihydro-3H-pyrrolo[3,2-d]pyrimidine-7-carboxamide (13)} A mixture of 3-amino-2-cyanopyrrole $4 \mathbf{a}(0.01 \mathrm{~mol})$ and formic acid $(25 \mathrm{~mL})$ was stirred at reflux temperature for $8 \mathrm{~h}$. The reaction mixture was then allowed to cool, poured onto crushed 
ice (50 g), neutralized with sodium hydroxide solution $(2 \mathrm{~N})$, filtered, dried, and crystallized from a mixture of EtOH-DMF, (2:1).

Yield (77\%); white solid, mp. 318-320 ${ }^{\circ} \mathrm{C}(\mathrm{EtOH}-\mathrm{DMF})$; IR (Nujol): $v=3376\left(\mathrm{NH}_{2}\right), 3179(\mathrm{NH})$ $\mathrm{cm}^{-1}$; ${ }^{1} \mathrm{H}$ NMR $\left(\mathrm{DMSO}_{6}\right): \delta=3.81\left(\mathrm{~s}, 3 \mathrm{H}, \mathrm{OCH}_{3}\right), 7.01$ (d, 2H, $\left.J=9.0 \mathrm{~Hz}, \mathrm{H}-3^{\prime}, 5^{\prime}\right), 7.44$ (d, $\left.2 \mathrm{H}, J=9.3 \mathrm{~Hz}, \mathrm{H}-2^{\prime}, 6^{\prime}\right), 7.48$ (s, 1H, NH, $\mathrm{D}_{2} \mathrm{O}$ exchange), 7.86 (s, 1H, NH, $\mathrm{D}_{2} \mathrm{O}$ exchange), 7.90 (s, 1H, H-2), 8.06 (s, 1H, H-6), 12.35 (s, 1H, NH, D $2 \mathrm{O}$ exchange). MS (EI, $70 \mathrm{eV}): \mathrm{m} / z(\%)$ = 285.09 (15); 284.09 (100, M+); 269.08 (12); 268.07 (65); 266.08 (100); 251.06 (32). Anal. Calcd. for $\mathrm{C}_{14} \mathrm{H}_{12} \mathrm{~N}_{4} \mathrm{O}_{3}$ (284.27): C, 59.15; H, 4.25; N, 19.71. Found: C, 59.26; H, 4.34; N, 19.31 .

\section{4-Chloro-5-(4-methoxyphenyl)-5H-pyrrolo[3,2-d]pyrimidine-7-carbonitrile (14)}

A mixture of pyrrolopyrimidin-4-one $13(0.01 \mathrm{~mol})$ and phosphorus oxychloride $(25 \mathrm{~mL})$ was refluxed for $9 \mathrm{~h}$. After the completion of reaction, the excess of phosphorus oxychloride was removed under vacuum. The cooled reaction mixture was then added to crushed ice $(25 \mathrm{~g})$. The resulting solid was filtered, washed with sodium bicarbonate $(5 \% \mathrm{w} / \mathrm{v})$ followed by cold water, dried, and crystallized from ethanol and chloroform (8:2 v $\backslash \mathrm{v}$ ). Yield (64\%); beige solid, mp. 206-208 ${ }^{\circ} \mathrm{C}(\mathrm{EtOH})$. IR (Nujol): $v=2230(\mathrm{CN}) \mathrm{cm}^{-1} ;{ }^{1} \mathrm{H}$ NMR (DMSO-d $): \delta=3.84$ (s, 3H, $\mathrm{OCH}_{3}$ ), 7.10 (d, 2H, $\left.J=9.0 \mathrm{~Hz}, \mathrm{H}-3^{\prime}, 5^{\prime}\right), 7.57$ (d, 2H, $\left.J=9.0 \mathrm{~Hz}, \mathrm{H}-2^{\prime}, 6^{\prime}\right), 8.89$ (s, 1H, H-2), 8.95 (s, 1H, H-6); ${ }^{13} \mathrm{C}$ NMR (DMSO-d $): \delta=55.59\left(\mathrm{OCH}_{3}\right), 86.87(\mathrm{C}-7), 113.18(\mathrm{CN}), 114.05$ (C-3`,5 ), 124.22 (C-7a), 129.24 (C-2`,6`), 129.33 (C-1 '), 143.46 (C-4a), 145.23 (C-6), 150.85 (C-4), 151.50 (C-2), 160.14 (C-4'). Anal. Calcd. for $\mathrm{C}_{14} \mathrm{H}_{9} \mathrm{ClN}_{4} \mathrm{O}{ }_{1}^{1 / 3} \mathrm{H}_{2} \mathrm{O} \mathrm{C}, 57.79 ; \mathrm{H}, 3.09 ; \mathrm{N}$, 19.26. Found: $58.00 ; 3.40 ; 18.96$.

\section{5-(4-Methoxyphenyl)-4-oxo-3-phenyl-4,5-dihydro-3H-pyrrolo[3,2-d]pyrimidine-7- carbonitrile (15)}

Method A. To a solution of amidine $7 \mathbf{b}(3.0 \mathrm{mmol})$ in methanol $(20 \mathrm{~mL})$ was added aniline $(3.0$ mmol). The reaction mixture was heated under reflux for $7 \mathrm{~h}$. The precipitate formed after cooling overnight was filtered off and dried and recrystallized from ethanol $(71 \%)$.

Method B. Aniline $(2.0 \mathrm{mmol})$ and $p$-toluenesulfonic acid $(0.01 \mathrm{~g})$ were added to a solution of amidine $\mathbf{8 b}(2.0 \mathrm{mmol})$ in toluene $(40 \mathrm{~mL})$. The reaction mixture was refluxed for $7 \mathrm{~h}$, and the precipitated solid was filtered off and crystallized from EtOH $(66 \%)$.

Yield (71 \%); white solid, mp. 258-260 ${ }^{\circ} \mathrm{C}$ (EtOH-DMF); IR (Nujol): $v=2232$ (CN), 1697 (CO) $\mathrm{cm}^{-1} ;{ }^{1} \mathrm{H}$ NMR $\left(\mathrm{DMSO}_{-}\right): \delta=3.79\left(\mathrm{~s}, 3 \mathrm{H}, \mathrm{OCH}_{3}\right), 7.00\left(\mathrm{~d}, 2 \mathrm{H}, J=8.7 \mathrm{~Hz}, \mathrm{H}-3^{\prime}, 5^{\prime}\right), 7.40-7.60$ $(\mathrm{m}, 7 \mathrm{H}, \mathrm{Ar}-\mathrm{H}), 8.35(\mathrm{~s}, 1 \mathrm{H}, \mathrm{H}-2), 8.50(\mathrm{~s}, 1 \mathrm{H}, \mathrm{H}-6) ;{ }^{13} \mathrm{C} \mathrm{NMR}\left(\mathrm{DMSO}-\mathrm{d}_{6}\right): \delta=55.52\left(\mathrm{OCH}_{3}\right)$, 87.86 (C-7), 113.66 (C-3',5'), 113.76 (C-7a), 117.02 (CN), 127.29 (C-2'", 6"), 127.72 (C-3"', $\left.5^{\prime \prime}\right), 128.86\left(\mathrm{C}-4^{\prime \prime}\right), 129.10\left(\mathrm{C}-2{ }^{\prime}, 6^{\prime}\right), 130.52$ (C-1'), $137.00\left(\mathrm{C}-1^{\prime \prime}\right), 138.41$ (C-6), 145.49 (C4a), 147.50 (C-2), $152.00(\mathrm{C}-4, \mathrm{C}=\mathrm{O}), 159.21$ (C-4). Anal. Calcd. for $\mathrm{C}_{20} \mathrm{H}_{14} \mathrm{~N}_{4} \mathrm{O}_{2} 1 / 6 \mathrm{H}_{2} \mathrm{O} \mathrm{C}$, 69.49; H, 4.05; N, 16.21. Found C, 69.26; 4.14; 16.11 . 


\section{Acknowledgements}

We thank Fundação para a Ciência e Tecnologia and FEDER (POCTI-SFA-3-686) and postDoctoral grant for A. Salaheldin (SFRH/BPD/31490/2006) and Miss Elisa Pinto for obtaining the NMR, and elemental analyses data.

\section{References}

1. Tarzia, G.; Panzone, G.; Leali, M.; Burdisso, M.; Schiatti, P.; Selva, D. Farmaco, Ed. Sci. 1984, 39, 538.

2. Tarzia, G.; Panzone, G. U.S. Patent 4,140,696, 1988.

3. Unverferth, K.; Engel, J.; Hoefgen, N.; Rostock, A.; Guenther, R. ; Lankau, H.-J.; Menzer, M.; Rolfs, A.; Liebscher, J.; Mueller, B.; Hofmann, H.-J. J. Med. Chem. 1998, 41, 63.

4. Otmar, M.; Masojídková M.; Votruba, I.; Holý. A. Bioorg. Med. Chem. 2004, 12, 3187.

5. Patterson, J. M. Synthesis 1976, 281.

6. Sundberg, R. J. In Comprehensive Heterocyclic Chemistry, Vol. 4, Katritzky, A. R.; Rees, C. W., Eds.; Pergamon Press: Oxford, 1984; pp 313-376.; Sundberg, R. J.; In Comprehensive Heterocyclic Chemistry II, Vol. 2 Katritzky, A. R.; Rees, C. W.; Scriven, E. F. V., Eds.; Pergamon Press: Oxford, 1996, pp 119.

7. Selič, L.; Stanovnik, B. Helv. Chem. Acta 1998, 81, 1634.

8. Barton, D. H. R.; Kervagoret, J.; Zard, S. Z. Tetrahedron 1990, 46, 7587.

9. Black, D. St.C. In Science of Synthesis, Vol. 9, Mass, G., Ed., Thieme: Stuttgart, 2000, pp 441.

10. Goncalves, M. S. T.; Oliveira-Campos, A. M. F.; Rodrigues, L. M.; Proenca, M. F. R. P.; Griffiths, J.; Maia, H. L. S.; Kaja, M.; Hrdina, R. J. Chem. Res. 2004, 3, 115.

11. Gupta S.; Rodrigues, L. M.; Esteves, A. P.; Oliveira-Campos, A. M. F.; Nascimento, M. S. J.; Nazareth, N.; Cidade, H.; Neves, M. P.; Pinto, E. F. M.; Cerqueira, N. M. F. and Brás N. Eur. J. Med. Chem. 2008, 43, 777.

12. Salaheldin, A. M.; Abdallah, T. A.; Radwan, N. F.; Hassaneen, H. M. Z. Naturforsch. 2006, $61 b, 1158$.

13. Salaheldin, A. M.; Oliveira-Campos, A. M. F.; Rodrigues, L. M. Tetrahedron Lett. 2007, 48, 8819.

14. Oliveira-Campos, A. M. F.; Salaheldin, A. M.; Rodrigues, L. M. Arkivoc 2007, (xvi), 92.

15. Gewald, K.; Schäfer, H.; Bellmann, P.; Hain, U. J. Prakt. Chem. 1992, 334, 491.

16. Rehwald, M.; Schäfer, H.; Gewald, K. Monatsh. Chem. 1997, 128, 933.

17. Lim, M.-I. ; Klein, R. S.; Fox, J. J. J. Org. Chem. 1979, 44, 3826.

18. Evans, G. B.; Furneaux, R. H.; Hutchison, T. L.; Kezar, H. S.; Morris, P. E.; Schramm, Jr. V. L.; Tyler, P. C. J. Org. Chem. 2001, 66, 5723.

19. Otmar, M.; Masoj1dkova, M.; Votruba, I.; Holy, A. Bioorg. \& Med. Chem. 2004, 12, 3187. 
20. Mitteilung, K.; Wolfbeis, O. S. Monatsch. Chemie 1981, 112, 875.

21. Amarnath, V.; Madhav, R. Synthesis 1974, 837.

22. Garcia-Lopez, M. T.; De las Heras, F. G.; Stud, M. J. Chem. Soc., Perkin Trans. 1, 1978, 483.

23. Dimroth Rearrangement. Translocation of heteroatoms in heterocyclic rings and its role in ring transformations of heterocycles, El Ashry, E. S. H.; El Kilany, Y.; Rashed, N.; Assafir, H. In Advances Heterocyclic Chemistry, Katritziky, A. R., Ed.; Academic Press: New York, 1999; Vol. 75, p 79.

24. Cheng, C. C.; Robins, R. K. J. Org. Chem. 1956, 21, 1240. 